\title{
КОНТРОЛЬ ДЕФЕКТНОСТИ ИЗОЛЯЦИИ ОБМОТОЧНЫХ ПРОВОДОВ - ЭФФЕКТИВНЫЙ ПРОЦЕСС ПОВЫШЕНИЯ НАДЁЖНОСТИ ЭЛЕКТРОДВИГАТЕЛЕЙ ГОРНОДОБЫВАЮЩЕЙ, СТРОИТЕЛЬНОЙ И ИНЫХ СФЕР ЧЕЛОВЕЧЕСКОЙ ДЕЯТЕЛЬНОСТИ
}

\author{
Смирнов Геннадий Васильевич, \\ smirnov@main.tusur.ru \\ Томский государственный университет систем управления и радиоэлектроники, \\ Россия, 634045, г. Томск пр. Ленина, 40.
}

\begin{abstract}
Актуальность работы. Такие сферы деятельности людей, как геологическая разведка, добыча, перевозка и переработка добытого сырья используют средства механизации и автоматизации с применением разных типов электрических приводов. Показатели надежности этих устройств определяются качеством изоляции обмоточных проводов. В связи с этим создание эфффективных средств контроля эмалевой изоляции проводов, улучшение качества этой изоляции, устранение технологических причин возникновения в ней дефектов являются востребованными и злободневными.

Цель: исследование влияния параметров измерителя дефектности, режимов процесса контроля и протекающих в первичном преобразователе дефектов газоразрядных процессов на точность измерения геометрических размеров дефектов в изоляции проводов.

Методы: осциллографический, микрометрический, теория планирования экспериментов, методы корреляционных оценок между параметрами контроля и регрессионный анализ.

Результаты. Выявлены и изложены закономерности возникновения систематической погрешности измерения протяженности дефектов при контроле изоляции обмоточных проводов в процессе намотки обмоток измерителем десектности, использующим в качестве первичного преобразователя дефектов газоразрядный датчик. Показано, что основное влияние на величину указанной погрешности оказывают два фактора: напряжение контроля $U$ на газоразрядном датчике дефектов и постоянная времени $\tau=R C$ разрядной цепи датчика. С использованием корреляционного и регрессионного анализов построена адекватная модель систематической погрешности и установлено, что скорость движения провода не оказывает существенного влияния на величину этой погрешности. С применением теории планирования экспериментов получено адекватное уравнение зависимости систематической погрешности от параметров контроля. Показано практическое применение установленных закономерностей для повышения точности контроля протяженности дефектов в изоляции провода в измерителях дефектности с газоразрядным датчиком.
\end{abstract}

\section{Ключевые слова:}

Контроль, дефект, протяженность, первичный преобразователь дефектов, погрешность, датчик скорости, напряжение, постоянная времени, низковольтная обмотка, коронный разряд.

\section{Введение}

ГОСТ IEC 60851-5-2011 [1] предусматривает контроль изоляции проводов на наличие в них дефектных участков.

Этому стандарту присущ ряд недостатков: сменность датчика дефектов при переходе от контроля проводов одного диаметра к другому; возникновение при контроле за счет перегибов и трения о поверхности роликов-электродов механических нагрузок, приводящих к дополнительному дефектообразованию в изоляции; требование постоянства скорости протягивания провода через первичный преобразователь, что делает невозможным осуществлять контроль при высоких или переменных скоростях его перемещения; низкая информативность и достоверность контроля, что связано с выборочностью контроля относительно малых участков изоляции провода в контролируемой катушке.

Ещё одним значительным недостатком упомянутого выше стандарта является отсутствие возможности определения протяженности дефектов, которая не менее важна для оценки качественных показателей изоляции, чем количество дефектов [2, 3].

Перечисленные недостатки существенно ограничивают возможности контроля, и его можно приме- нять только для выборочного входного или выходного контроля изоляции провода.

На наш взгляд, наиболее целесообразно проводить сплошной технологический контроль дефектности там, где используется преобладающее количество выпускаемого провода, в частности в производственных процессах изготовления обмоток электрических машин, потому что именно в этих процессах образуется наибольшее количество дефектов. Применение сплошного контроля при производстве электрических машин может дать значительный экономический эффект, так как этот вид электротехнической продукции пользуется высоким спросом во всех сферах деятельности людей и является продуктом массового производства. При таком массовом выпуске даже небольшое снижение производственного брака обмоток и их эксплуатационных отказов, к чему может привести правильная организация контроля, может дать значительный экономический эффект. О распространенности электрических машин можно судить, например, по их применимости более чем в 85 \% всех транспортных и производственных агрегатах [3-8].

Стоимость изоляции электродвигателя равна примерно пятидесяти процентам стоимости остальных элементов машины, в связи с чем весьма важным 
процессом представляется диагностика состояния изоляции при её изготовлении и оперативное устранение причин, приводящих к падению качества изоляции. Причинами отказов электродвигателей являются тяжелые условия их эксплуатации и нередко плохое их обслуживание. Статистика говорит о том, что 30-35 \% отказов обусловлены неудовлетворительной конструкцией и несовершенством технологии изготовления двигателей. Примерно около 10-12 \% отказов электрических машин происходит в результате естественных процессов износа и старения изоляционных материалов машины. По статистике около 85-95 \% от общего числа отказов электродвигателей происходит по причине отказа изоляции статорной обмотки [9] При этом отказы из-за витковых замыканий составляют $93 \%$, из-за выхода из строя фазовой изоляции - $5 \%$, а пазовой изоляции - всего лишь $2 \%$ [3].

Аварийный отказ электропривода может привести к значительным убыткам предприятия, связанным не только с необходимостью замены отказавшей машины, но и с возникшим из-за отказа простоем производственного оборудования. Ущерб от простоя может значительно превышать стоимость отказавшего механизма.

Уровень аварийности электроприводов неравномерно распределен по областям их применения. Так, в устройствах, связанных с добычей горных руд ежегодно наблюдается около 30 \% отказов. В других отраслях этот процент колеблется от $10 \%$ у производств в металлургии до 55 \% в строительстве. Относительно невысокий срок службы у двигателей, работающих в шахтах, который составлял, например, на отдельных шахтах Кузбасса всего около 6-7 месяцев.

Как показывает практика, преобладающее число повреждений витковой изоляции обмоток возникает при их намотке и всыпки в пазы статоров $[10,11]$.

При этом производственный брак обмоток из-за возникновения витковых, межфазных или корпусных замыканий, процент отказов обмоток при их приработке и эксплуатации зависят не только от количества этих дефектов, но и от их геометрических размеров. Без контроля изоляции обмоточных проводов в процессе производства из них обмоток невозможно выявить и оперативно устранить эти причины. Существенным вкладом в этом направлении являются работы $[12,13]$, в которых описан способ контроля изоляции проводов с применением газоразрядного первичного преобразователя [13, 14]. Газоразрядный датчик дефектов очень чувствителен и позволяет контролировать дефектность изоляции провода при высоких и переменных скоростях движения провода. Однако до сих пор остаётся открытым вопрос, насколько приемлем предложенный контроль для определения не только количества дефектов в изоляции проводов, но и не менее важной характеристики геометрических размеров (протяженностей) этих дефектов. Нет и достаточного четкого обоснования того, как влияет скорость движения провода, напряжение и параметры разрядной цепи первичного преобразователя дефектов на погрешность определения указан- ной характеристики - протяженности дефектов. Попытка ответить на эти вопросы и предпринята в настоящей статье.

\section{Обоснование и описание методики эксперимента}

При использовании газоразрядного датчика дефектов после выполнения условия самостоятельности зажигания могут возникнуть различные виды разряда: коронный, тлеющий, искровой и дуговой. Кроме того, поскольку во время контроля дефектности изолированный провод приближается к датчику, а в процессе этого перемещения роль подвижного электрода играет жила провода в месте дефекта (повреждения изоляции до жилы провода), то на определенном расстоянии от датчика могут создаться условия для инициирования разряда по поверхности диэлектрика - изоляции провода. При прохождении дефектного участка под датчиком этот поверхностный разряд может изменится и перейти в любой другой вид разряда, из указанных выше. При проходе дефекта области датчика и удалении от датчика зажжённый разряд может еще продолжаться некоторое время, а потом гаснет. Характер разряда, возникающего при прохождении дефекта в зоне действия датчика, как правило, сопровождается изменением напряжения на датчике и протеканием тока в разрядном промежутке. Эти изменяющиеся параметры в разрядной цепи датчика являются носителями информации о виде разряда, количестве дефектов на изоляции провода и их протяженности. При этом следует учесть, что если один и тот же дефект протягивать через датчик дефектов, создавая на датчике и в разрядной цепи различные условия, то под действием этих изменяющихся условий информационные характеристики, напряжение на датчике и ток через него тоже будут изменяться. Рассмотрим, используя осциллографический метод, как влияют на эти изменяющиеся параметры различные факторы контроля и как можно извлечь полезную информацию о количестве и протяженности дефектов из изменяющихся параметров разряда.

Из априорной информации известно, вид разряда в значительной мере зависит от параметров разрядной цепи датчика, мощности источника напряжения на датчике, уровня и полярности напряжения на нём.

Из перечисленных выше видов разряда не приемлемым является дуговой, так как он сопровождается большой плотностью тока и интенсивным разрушением электродов. Поэтому исключить данный вид разряда можно уменьшением мощности источника напряжения или уменьшением тока в разрядной цепи. В свою очередь ток в разрядной цепи можно ограничить увеличением сопротивления этой цепи.

При протягивании провода по мере приближения дефектного участка к датчику дефектов между ними при определенном расстоянии могут создаваться условия, необходимые для зажигания коронного разряда, который при уменьшении расстояния между датчиком и дефектом с некоторой вероятностью может перейти в иной вид разряда. Отличительной особенностью коронного разряда является то, что ток 
разряда мал и не зависит от параметров внешней цепи, а определяется проводимостью внешней, не коронирующей областью разряда. Устойчивый коронный разряд возникает в случае, если размер электрода, имеющего большую кривизну, гораздо меньше межэлектродного расстояния. При контроле дефектов в изоляции движущегося провода разряд зажигается при нестабильном, динамически изменяющемся межэлектродном расстоянии. Помимо этого, возникновение условий для зажигания самостоятельного разряда зависит от поверхности эмалевой изоляции, формы и протяженности дефекта, продольных колебаний провода и других факторов. В связи с этим разряд нестабилен и по мере приближения дефекта к датчику переходит в один из других видов разряда.

На рис. 1 приведена схема электрической цепи разрядного промежутка первичного газоразрядного преобразователя.

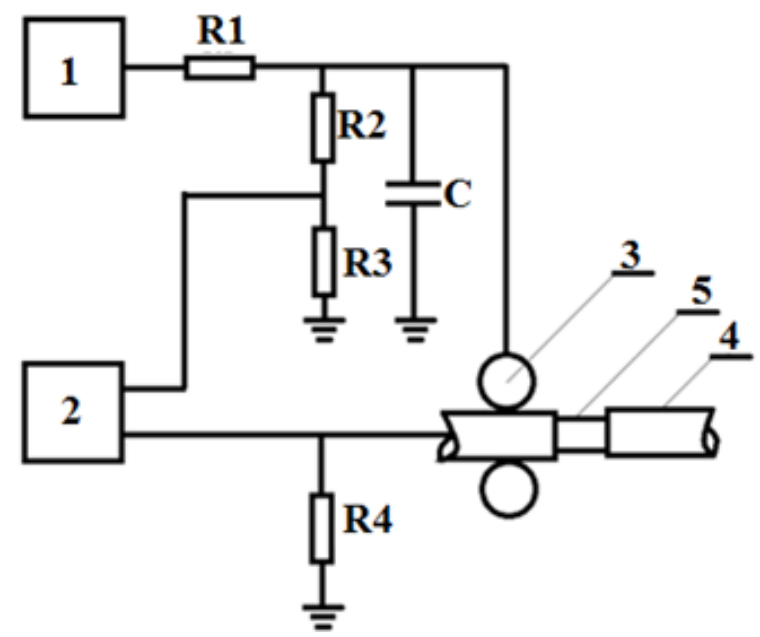

Рис. 1. Схема электрической чепи разрядного промежутка: 1- высоковольтный источник напряжения (ВИН); 2 - осииллограф; 3 - ролик датчика дефектов; 4 - изоляция контролируемого провода; 5 - дефект

Fig. 1. Diagram of the electrical circuit of the discharge gap: 1- high voltage source (HVS); 2 -oscilloscope; 3 - sensor defect roller; 4 - insulation of the controlled wire; 5-defect

Основными параметрами схемы на рис. 1 являются разрядные сопротивление $\mathrm{R} 1$ и ёмкость $C$. Аналогичная схема разрядной цепи датчика была использована в экспериментах работы [14]. Подробный анализ осциллограмм, проведенных в этой работе, показал, что у загоревшегося разряда при прохождении через датчик дефектного участка изоляции напряжение и ток разряда непрерывно изменяются и носят явно выраженный импульсный характер. Такой характер тока и напряжения связан с тем, что ёмкость $C$ при возникновении коронного разряда начинает разряжаться, ток разряда по экспоненте возрастает, а напряжение по экспоненте падает.

При падении напряжения напряженность поля в области дефекта снижается до некоторой величины, при которой разряд гаснет. После погасания разряда воздушный разрядный промежуток восстанавливает свою электрическую прочность. Одновременно с восстановлением электрической прочности промежутка происходит подзарядка ёмкости $C$ протекающим током разряда, и напряжение на датчике начинает возрастать. После достижения на разрядном промежутке условий, необходимых для зажигания самостоятельного разряда, вновь возникает искра и процесс повторяется.

При смене ёмкости $C$ разрядной цепи характер осциллограмм изменяется.

Фронты импульсов тока и напряжения, как это следует из осциллограмм, приведенных в работе [14], изменяются по экспоненте и зависят от постоянной времени, равной $\tau=R_{1} C$. С уменьшением постоянной времени снижается время заряда ёмкости $C$ и частота разрядных импульсов увеличивается. При меньшем значении $\tau$ продолжительность импульсов тока и импульсов пульсаций напряжения относительно мала и возрастает с увеличением $\tau$. При уменьшении $\tau$ до значений, при которых скорость восстановления электрической прочности разрядного промежутка становится меньше скорости нарастания напряжения на ёмкости $C$, повторные зажигания искр происходят при меньших напряжениях.

Характеристики сигнала дефекта с первичного преобразователя при положительной полярности напряжения на нём незначительно отличаются от характеристик сигнала при отрицательной полярности потенциала. Возможно, такое изменение сигнала связано с тем, что при отрицательном потенциале на датчике процесс образования лавин идет более интенсивно, так как электрод с большей кривизной, роль которого выполняет жила провода в области дефекта, является анодом. Электронные лавины, двигаясь к аноду, оставляют после себя положительные ионы [15-17].

Скорость движения этих положительных ионов на два порядка меньше скорости перемещения электронов. Вблизи анода происходит накопление положительных ионов, и анод как бы удлиняется, и напряженность электрического поля в области анода возрастает. Осциллографические исследования показали, что если один и тот же дефектный участок строго фиксированной протяженностью, например 0,5 мм, многократно протягивать с одной и той же скоростью через датчик дефектов, то в сигнале с первичного преобразователя (датчика) дефектов можно выделить две области.

Одна из них является относительно стабильной (на осциллограмме в работе [14] она обозначена $t_{c}$ ). Другая область является нестабильной и обозначена на упомянутых осциллограммах как $t_{p}$. Время $t_{p}-$ это время всего сигнала с первичного преобразователя при прохождении через него одного дефекта. Оно включает в себя время горения всего разряда между первой и последней искрами, возникшими при прохождении дефектного участка в зоне реакции датчика дефектов. Наличие этих двух зон можно объяснить следующим образом. При входе дефектного участка в 
зону действия первичного преобразователя и при выходе этого участка из упомянутой зоны возникающий разряд нестабилен. Это связано с неизбежными флуктуациями достижения условий самостоятельности зажигания разряда, которое может выполняться при различных расстояниях между дефектом и датчиком. В зоне нестабильности повторные зажигания разряда могут произойти не сразу после восстановления напряжения на датчике дефектов до начального значения, а с задержкой, по мере приближения дефекта к датчику.

Аналогичная ситуация происходит и при выходе дефектного участка из зоны действия датчика. Количество пауз и их длительность весьма нестабильны и могут изменяться в широких пределах не только от дефекта к дефекту, но и при многократном протягивании одного и того же дефекта через датчик. При приближении дефекта к датчику и нарастании напряженности электрического поля до некоторой критической величины разряд стабилизируется.

Наличие стабильной зоны в сигнале первичного преобразователя дефектов дало основание предположить, что именно из этой зоны можно извлечь информацию о протяженности дефектного участка изоляции. Первоначально было высказано предположение о том, что время $t_{c i}$ зависит не только от протяженности дефекта, но и от напряжения $U_{0}$ на датчике дефектов, значений $C$ и $R 1$ в разрядной цепи, а также от скорости перемещения провода $V$ относительно датчика дефектов. Проверка этой гипотезы осуществлялась на специально разработанной и созданной для этой цели экспериментальной установке. В установку входил диск из диэлектрического материала диаметром 600 мм, вращаемый через редуктор двигателем постоянного тока. По образующей поверхности диска была проточена канавка глубиной $h=0,3$ мм, в которую укладывался контролируемый провод диаметром $d=0,8$ мм, с нанесенным на его изоляции кольцевым дефектом протяженностью $l_{\text {д }}=0,5$ мм. Концы провода были выведены на боковую поверхность диска и электрически соединены с металлической осью, на которую был закреплен диск. Ось через скользящий контакт и резистор, являющийся датчиком тока, была заземлена. Регулировка скорости вращения диска осуществлялась изменением напряжения питания двигателя. Скорость вращения диска $V_{1}$ контролировалась тахометром ТЕМП-4. К поверхности контролируемого провода, уложенного в проточку на образующей части диска, прижимался подпружиненный, свободно вращающийся ролик из нержавеющей стали диаметром 13 мм. На ось ролика через скользящий контакт подавалось напряжение контроля. При частоте вращения диска $v$ скорость движения провода $V$ определялась из выражения:

$$
V=v \times \pi \times(D+d-h),
$$

где $v$ - частота вращения диска об/с; $D$ - диаметр диска; $d$ - диаметр провода; $h$ - глубина проточки.

Определяемая с использованием осциллограммы

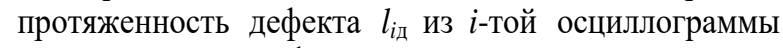
рассчитывалась по формуле:

$$
l_{i д}=V \times t_{i c} .
$$

В экспериментах варьировались следующие факторы: напряжение $U$ на датчике, сопротивление $R 1$, ёмкость $C$ и скорость $V$ движения провода.

Выбор диапазонов изменения варьируемых факторов основывался на следующих соображениях.

Напряжение на датчике дефектов изменялось в диапазоне от 0,5 до 2 кВ. При напряжениях ниже 0,5 кВ время $t_{i c}$ в осциллограммах, полученных при прохождении через датчик дефектов одного и того же дефектного участка неизменного по длине поврежденного участка изоляции провода, при многократном повторении изменялось. Вероятно, это связано с тем, что условия зажигания самостоятельного разряда в данном случае также изменялись от одного протягивания дефекта к другому, что в значительной мере зависело от продольных колебаний провода, состояния поверхности и других факторов. Иногда разряд при данном напряжении мог и не зажигаться. Еще большая нестабильность наблюдалась при напряжениях на датчике больше 2 кВ. Это связано с тем, что при подходе переднего фронта дефектного участка к датчику дефектов разряд мог зажигаться, а затем гаснуть на значительном расстоянии от первичного преобразователя несколько раз. Такое явление могло происходить до тех пор, пока между жилой провода в дефектном участке и датчиком дефектов не зажигался стабильный разряд. Аналогичная картина наблюдалась и при выходе дефектного участка из зоны действия датчика.

Стремление повысить стабильность зоны $t_{i c}$ заставляет уменьшить постоянную времени $\tau$ заряда ёмкости $C$. Однако с уменьшением сопротивления $R 1$ увеличивается плотность тока, протекающего через разрядный канал, что приводит к увеличению вероятности смены вида разряда. О смене вида разряда свидетельствует перераспределение яркости свечения разрядного канала, слабое свечение которого переходит к резкому свечению в прикатодной области. В момент смены вида разряда напряжение на датчике дефектов резко падает до значений (400...600) В и стабилизируется. Стабилизируется и ток, протекающий через разрядный промежуток. Предположительно, происходит зажигание тлеющего разряда, так как описанные выше признаки и характеристики присущи тлеющему разряду. Возможность зажигания тлеющего разряда в воздухе при атмосферном давлении рассмотрена в работах [17-19]. Напряжение на первичном преобразователе при зажигании тлеющего разряда равно падению напряжения катодного слоя $(U \approx 300$ В [17]) и падению напряжения на положительном столбе. При удалении дефекта от датчика увеличивается длина положительного столба и падения напряжения на нём. Напряжение на датчике возрастает.

При атмосферном давлении в воздухе тлеющий разряд нестабилен и под влиянием различных факторов за счет контракции положительного столба или катодного слоя переходит в искровой [19-24] или гаснет, если в момент контракции расстояние между 
дефектом и датчиком принимает значение, при котором не выполняется условие зажигания самостоятельного разряда. При зажигании тлеющего разряда время $t_{c i}$ резко возрастает и увеличивается его нестабильность.

Аналогичная картина наблюдается и при смене полярности напряжения на датчике дефектов.

При увеличении $R 1$ и $C$ возрастает величина $t_{c i}$ При значениях $R 1>3$ МОм и $C>240$ пФ возрастает нестабильность разряда, что приводит к росту погрешности в определении протяженности дефектов по длительности $t_{c i}$. Исходя из приведенных фактов, сопротивление и ёмкость в опытах изменялись в диапазонах: $C=(20 \ldots 240$ пФ), а $R l=(300$ кОм...3 МОм).

Выбор диапазона движения скоростей провода был основан на том, что разрабатываемую систему контроля изоляции проводов предполагалось использовать не только для выборочного контроля, как это рекомендовано ГОСТ [1], но и непосредственно в технологических процессах эмалирования проводов, а также в процессах изготовления из них обмоток электротехнических изделий. Предварительные измерения, проведенные на различных намоточных станках, показали, что максимальная скорость движения провода при изготовлении обмоток статоров электродвигателей не превышает 10 м/с. Поэтому в дальнейших опытах ограничивались указанной скоростью. В опытах скорости определялись по выражению (1) и варьировались, принимая следующие значения: 1, 3, 5, 7,9 и $10 \mathrm{~m} / \mathrm{c}$.

Величины ёмкостей разрядного промежутка и токоограничительных сопротивлений изменялись от опыта к опыту в диапазонах от 20 до 240 пФ и от 300 кОм до 3 МОм, соответственно. С высоковольтного источника напряжение положительной или отрицательной полярности через сменные токоограничительные резисторы подавалось на ролик датчика дефектов. Напряжения на ролики датчика дефектов изменяли и устанавливали равными: 0,$5 ; 0,8 ; 1,2 ; 1,8$; 2 кВ. При различных комбинациях $R l, C$ и $V$ и напряжениях контроля $U$ снималось по 200 осциллограмм, из которых определялось время $t_{c i}$. По полученным результатам рассчитывался коэффициент корреляции по формуле:

$$
r^{*}=\frac{\sum_{i=1}^{N}\left(x_{i}-\bar{x}\right)\left(y_{i}-\bar{y}\right)}{(N-1) S_{x} S_{y}},
$$

где $S_{x}$ и $S_{y}$ - выборочные среднеквадратичные отклонения исследуемых параметров.

Коэффициенты корреляции по выборке 56 опытов между $\bar{l}_{c 1}$ и факторами $U, C, R l, V$ рассчитывались с использованием редактора Excel и дали следующие результаты: $r_{l U}=0,887 ; r_{l c}=0,363 ; r_{l R}=0,609 ; r_{l V}=0,231$; $r_{l R C}=0,687$.

По шкале Чеддока [25] полученные результаты показали, что между протяженностью дефекта $\bar{l}_{c 1}$, определенной из осциллограмм по формуле (2), и напряжением $U$ на первичном преобразователе связь высокая $\left(r_{l U}=0,887\right)$. Связь между протяженностью дефекта $l_{\text {iд }}$, определенной из осциллограмм по формуле (2), сопротивлением $R l\left(r_{l R}=0,609\right)$ и постоянной времени $\tau=C \times R 1\left(r_{\Delta l R C}=0,687\right)$ заметная, связь между протяженностью дефекта $\bar{l}_{c 1}$, определенной из осциллограмм по формуле $(2)$, и емкостью $C\left(r_{l c}=0,363\right)$ умеренная, а связь со скоростью провода $V\left(r_{l V}=0,231\right)$ слабая.

Практическое отсутствие влияния скорости движения провода на длительность импульса дефекта можно объяснить тем, что при приближении дефектного участка изоляции провода к датчику дефектов и достижении в области дефекта условий, необходимых для зажигания разряда, время запаздывания разряда так мало и процесс загорания разряда с этого момента происходит столь интенсивно и быстро, что при контроле изоляции провод, движущийся с любыми скоростями, которые могут быть реализованы в технологических процессах намотки обмоток, за это время может пройти пренебрежительно малый отрезок пути l. Сказанное выше подтверждают следующие численные оценки. Известно, что скорость движения провода при намотке обмоток может изменятся в широких пределах и достигать величины $10 \mathrm{~m} / \mathrm{c}=10^{5} \mathrm{~mm} / \mathrm{c}$. Даже при такой высокой скорости за время развития разряда, имеющего порядок $t=10^{-7} \mathrm{c}$, провод переместится всего на величину $l=V \times t=10^{5} \times 10^{-7}=10^{-2}$ мм. Пренебрежительно малый отрезок $l$ не оказывает никакого влияния на измеряемую величину $l_{\text {iд. }}$ Выявленный факт является очень важным, поскольку он указывает на то, что применение первичного газоразрядного преобразователя не влияет на точность контроля и позволяет определять дефектность провода и протяженность дефектов с неизменной погрешностью не зависимо от того, с какой скоростью движется провод.

Корреляционный анализ показал, что влияние скорости движения провода при контроле весьма слабое, т. е. практически отсутствует. Поэтому этим фактором при планировании экспериментов на первом этапе было решено пренебречь. Остальные три фактора $U, C, R 1$ варьировались в соответствии с табл. 1.

Хотя корреляционный анализ показал, что влияние скорости на исследуемый параметр $\bar{y}_{i}=l_{i_{д}}$ пренебрежительно мало, во избежание возможных дополнительных погрешностей, вызванных этим фактором, опыты проводились при постоянной скорости движения провода, равной $V=1$ м/с.

Было решено определить зависимость систематической погрешности в определении протяженности дефектного участка изоляции от всех влияющих на этот параметр факторов с использованием полного факторного эксперимента. При этом в качестве функции отклика была выбрана величина протяженности дефекта $l_{\text {iд, }}$ определяемая по длительности стабильной зоны горения разряда по формуле (2).

В качестве уравнения регрессии была принята квадратичная модель, имеющая вид:

$$
\begin{aligned}
y= & b_{0}+b_{1} x_{1}+b_{2} x_{2}+b_{3} x_{3}+b_{11} x_{1}^{2}+b_{22} x_{2}^{2}+b_{33} x_{3}^{2}+ \\
& +b_{12} x_{1} x_{2}+b_{13} x_{1} x_{3}+b_{23} x_{2} x_{3}+b_{123} x_{1} x_{2} x_{3} .
\end{aligned}
$$


Для построения модели были использованы планы типа $B_{n}$. Планами типа $B_{n}$ называются композиционные планы с «ядром» в виде полного $\left(2^{n}\right)$ факторного плана, дополненного $2 n$ звездными точками с «плечом» $\alpha=1$. Иными словами, план типа $B_{n}$ включает в себя $2^{n}$ вершин $n$-мерного гиперкуба с координатами \pm 1 и $2 n$ имеющихся центров $(n-1)$-мерных граней. Общее количество точек плана $N=2^{n}+2 n$. Планы типа $\mathrm{B}_{\mathrm{n}}$ по такому важному критерию, как максимальное значение дисперсии предсказания $d_{\max }$, ненамного уступают непрерывным $D$-оптимальным планам и существенно превосходят ОЦКП и РЦКП Бокса (при меньшем числе опытов $N$ ). Дополнительное преимущество планов типа $B_{n}$ состоит в том, что их звездные точки находятся в пределах заданной области эксперимента $\Omega$, а не выходят за неё, что часто бывает невозможно осуществить.

Значения и диапазоны изменения влияющих на процесс контроля факторов приведены в табл. 1

Таблица 1. Значения и диапазоны изменения влияющих на процесс контроля факторов

Table 1. Values and ranges of changes affecting the control factors

\begin{tabular}{|c|c|c|c|c|c|c|}
\hline \multirow{2}{*}{$\begin{array}{l}\text { Фактор } \\
\text { Factor }\end{array}$} & \multicolumn{2}{|c|}{$\begin{array}{c}\text { Используемые } \\
\text { символы } \\
\text { Used symbols }\end{array}$} & \multicolumn{3}{|c|}{$\begin{array}{c}\text { Диапазон } \\
\text { варьирования } \\
\text { Variation range }\end{array}$} & \multirow{2}{*}{ 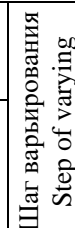 } \\
\hline & $\begin{array}{l}\text { действи- } \\
\text { тельное } \\
\text { valid }\end{array}$ & $\begin{array}{c}\text { кодовое } \\
\text { codeword }\end{array}$ & -1 & 0 & +1 & \\
\hline $\begin{array}{l}\text { Сопротивле- } \\
\text { ние, кОм } \\
\text { Resistance, } \\
\text { kOm }\end{array}$ & R1 & $\mathrm{X} 1$ & 400 & 650 & 900 & 250 \\
\hline $\begin{array}{l}\text { Емкость, пФ } \\
\text { Capacity, pF }\end{array}$ & C & $\mathrm{X} 2$ & 50 & 100 & 150 & 50 \\
\hline $\begin{array}{l}\text { Hапряжение, B } \\
\text { Voltage, V }\end{array}$ & $\mathrm{U}$ & X3 & 600 & 1300 & 2000 & 700 \\
\hline
\end{tabular}

Матрица плана приведена в табл. 2.

Таблица 2. Матрица плана по измерению дефектности Table 2. Matrix on the measurement of defects

\begin{tabular}{|c|c|c|c|c|c|}
\hline $\begin{array}{c}\text { № опыта } \\
\text { Experiment no. }\end{array}$ & $\mathrm{X} 1$ & $\mathrm{X} 2$ & $\mathrm{X} 3$ & $\bar{y}_{i}=\bar{l}_{c 1 i}$ & $S_{i}^{2}$ \\
\hline 1 & -1 & -1 & -1 & 1,79 & 0,0324 \\
\hline 2 & -1 & -1 & 1 & 6,32 & 0,0784 \\
\hline 3 & -1 & 1 & -1 & 1,43 & 0,0361 \\
\hline 4 & -1 & 1 & 1 & 5,91 & 0,0324 \\
\hline 5 & 1 & -1 & -1 & 1,57 & 0,0256 \\
\hline 6 & 1 & -1 & 1 & 6,02 & 0,0289 \\
\hline 7 & 1 & 1 & -1 & 1,71 & 0,0361 \\
\hline 8 & 1 & 1 & 1 & 6,26 & 0,0324 \\
\hline 9 & 1 & 0 & 0 & 3,78 & 0,0081 \\
\hline 10 & -1 & 0 & 0 & 3,83 & 0,0081 \\
\hline 11 & 0 & 1 & 0 & 3,61 & 0,0049 \\
\hline 12 & 0 & -1 & 0 & 4,01 & 0,01 \\
\hline 13 & 0 & 0 & 1 & 5,93 & 0,0081 \\
\hline 14 & 0 & 0 & -1 & 1,64 & 0,0121 \\
\hline 15 & 0 & 0 & 0 & 3,91 & 0,0025 \\
\hline
\end{tabular}

В работе [11] получены формулы для оценок коэффициентов квадратичной модели и их дисперсий, имеющие вид:

$$
b_{0}=\frac{1}{2(n-1)}\left(\sum_{j=N_{0}+1}^{N} \bar{y}_{j}-\frac{1}{2^{n-1}} \sum_{j=1}^{N_{0}} \bar{y}_{j}\right),
$$

где $N_{0}=2^{n}$;

$$
\begin{gathered}
b_{j j}=\frac{1}{2} \sum_{j=N_{0}+1}^{N}\left(x_{i j}\right)^{2} \bar{y}_{j}-b_{0} ; \\
b_{j}=\frac{1}{2+2^{n}} \sum_{j=1}^{N_{0}} x_{i j} \bar{y}_{i} \\
b_{j k}=\frac{1}{2^{n}} \sum_{j=1}^{N_{0}} x_{i j} x_{k j} \bar{y}_{i} .
\end{gathered}
$$

\section{Результаты экспериментов и их обсуждение}

Коэффициенты в уравнении (3) рассчитывались для $\bar{l}_{c 1}$ при $V=1 \mathrm{M} / \mathrm{c}$ по приведенным выше формулам (4)-(7). Они оказались равными: $b_{0}=3,761875$; $b_{1}=0,011 ; b_{2}=0,039 ; b_{3}=1,801 ; b_{12}=0,14375 ; b_{13}=-0,00125$; $b_{23}=0,00625 ; b_{123}=0,01875 ; b_{11}=0,043125 ; b_{22}=0,048125$; $b_{33}=0,0231125$.

Для оценки значимости найденных коэффициентов и адекватности уравнения регрессии (3) необходимо было найти дисперсию воспроизводимости. Процедура определения этой величины состояла в следующем.

В каждой точке плана было проведено по три опыта. По результатам параллельных $m=3$ опытов в каждой точке плана рассчитывалась дисперсия $S_{i}^{2}$. Значения $S_{i}^{2}$ приведены в табл. 2. Оценку однородности дисперсий проводили по критерию Кохрена:

$$
G=\frac{S_{i \max }^{2}}{\sum_{i=1}^{N=15} S_{i}^{2}} .
$$

Как следует из табл. 2, максимальное значение выборочной дисперсии $S_{i \max }^{2}=0,0784$.

Численное значение критерия Кохрена было равно:

$$
G=\frac{S_{i \max }^{2}}{\sum_{i=1}^{N=15} S_{i}^{2}}=\frac{0,0784}{0,3561}=0,22 .
$$

Табличное значение критерия Кохрена для $N=15$; $m=3$ при $5 \%$-м уровне значимости $G_{0,05}(N=15$; $m=3)=0,275$. Так как расчетное значение критерия Кохрена меньше табличного, т. е. выполняется неравенство $G<G_{0,05}(N=15 ; m=3)$, дисперсии однородны. Дисперсия воспроизводимости будет равна:

$$
S_{\text {воспр }}^{2}=\frac{\sum_{i=1}^{N=15} S_{i}^{2}}{15}=\frac{0,3561}{15}=0,02374 .
$$

Оценим значимость коэффициентов регрессии. Для этого рассчитаем дисперсии коэффициентов регрессии $D b_{0}, D b_{i}, D b_{i j}$ и $D b_{i i}$ по формулам [11]:

$$
\begin{aligned}
& D b_{0}=\frac{c_{1} S_{\text {воспр }}^{2}}{n} ; \\
& D b_{i}=\frac{S_{\text {воспр }}^{2}}{n\left(2+2^{n}\right)} ;
\end{aligned}
$$




$$
\begin{gathered}
D b_{i j}=\frac{S_{\text {воспр }}^{2}}{n 2^{n}} ; \\
D b_{i i}=\frac{c_{2} S_{\text {воспр }}^{2}}{n} .
\end{gathered}
$$

В формулах (8)-(11) величины $c_{1}$ и $c_{2}$ рассчитываются по выражениям:

$$
\begin{gathered}
c_{1}=\frac{2+n 2^{n}}{(n-1)^{2} 2^{n+1}} ; \\
c_{2}=\frac{\left\{[n(n-3)+3] 2^{n}+2\right\}}{(n-1)^{2} 2^{n+1}} .
\end{gathered}
$$

Численные значения этих величин, рассчитанные по формулам (12) и (13) будут равны:

$$
\begin{gathered}
c_{1}=\frac{2+n 2^{n}}{(n-1)^{2} 2^{n+1}}=\frac{26}{64}=0,40625 ; \\
c_{2}=\frac{\left\{[n(n-3)+3] 2^{n}+2\right\}}{(n-1)^{2} 2^{n+1}}=\frac{26}{64}=0,40625 .
\end{gathered}
$$

Рассчитываем критерий Стьюдента [11] по выражениям:

$$
t_{0}=\frac{\left|b_{0}\right|}{\sqrt{D b_{0}}} ; t_{i i}=\frac{\left|b_{i i}\right|}{\sqrt{D b_{i i}}} ; t_{i}=\frac{\left|b_{i}\right|}{\sqrt{D b_{i}}} ; t_{i j}=\frac{\left|b_{i j}\right|}{\sqrt{D b_{i j}}} .
$$

$t_{0}=67,5 ; t_{1}=0,39 ; t_{2}=1,39 ; t_{3}=64,32 ; t_{12}=4,63 ; t_{13}=0,04$;

$t_{23}=0,363 ; t_{123}=0,6 ; t_{11}=0,27 ; t_{22}=0,86 ; t_{33}=0,413$.

Критерий Стьюдента для числа степеней свободы $N(m-1)=14 \times 2=28$ равен $t_{0,05}[N(m-1)=28]=2,05$ [11].

Таким образом, значимыми коэффициентами являются только $b_{0}=3,761875 ; b_{3}=1,801 ; b_{12}=0,14375$.

Незначимые коэффициенты исключаем из уравнения. Окончательный вид уравнения регрессии будет

$$
y^{*}=3,76+1,801 x_{3}+0,14375 x_{1} x_{2} .
$$

Уравнение (14) показывает, что величина протяжённости дефектного участка изоляции, определяемая по длительности стабильной зоны разряда, не зависит от квадратичных эффектов, а определяется линейной зависимостью от уровня напряжения на датчике дефектов и постоянной времени, равной

$$
\tau=R 1 \times C \text {. }
$$

Для более точного описания модели изменим варьируемые факторы. Будем варьировать напряжение $U$ на датчике и постоянную времени $\tau$. Используем табл. 1 и формулу (15) для пересчета уровней варьи-

\begin{tabular}{|c|c|c|c|c|c|c|}
\hline \multirow[b]{2}{*}{$\begin{array}{l}\text { Фактор } \\
\text { Factor }\end{array}$} & \multicolumn{2}{|c|}{$\begin{array}{l}\text { Обозначение } \\
\text { Designation }\end{array}$} & \multicolumn{3}{|c|}{$\begin{array}{c}\text { Уровни } \\
\text { варьирования } \\
\text { Variation levels } \\
\end{array}$} & \multirow{2}{*}{ 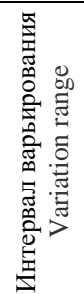 } \\
\hline & $\begin{array}{c}\text { действи- } \\
\text { тельное } \\
\text { valid }\end{array}$ & $\begin{array}{l}\text { кодовое } \\
\text { codeword }\end{array}$ & -1 & 0 & +1 & \\
\hline $\begin{array}{l}\text { Напряже- } \\
\text { ние, B } \\
\text { Voltage, V }\end{array}$ & $\mathrm{U}$ & $\mathrm{X} 1$ & 600 & 1300 & 2000 & 700 \\
\hline $\begin{array}{l}\text { Постоянная } \\
\text { времени, } \\
\text { мкс } \\
\text { Time } \\
\text { constant, } \\
\text { mks }\end{array}$ & $\tau=\mathrm{R}_{1} \mathrm{C}$ & $\mathrm{X} 2$ & 0,2 & 0,775 & 1,35 & 0,575 \\
\hline
\end{tabular}
руемой величины $\tau$.

$$
\begin{gathered}
\tau_{\min }=R 1_{\min } \times C_{\min }=400 \times 10^{3} \times 50 \times 10^{-12}=2 \times 10^{-5} \mathrm{c}=0,2 \mathrm{м \kappa с.} . \\
\tau_{\max }=R 1_{\max } \times C_{\max }= \\
=900 \times 10^{3} \times 150 \times 10^{-12}=1,35 \times 10^{-6} \mathrm{c}=1,35 \mathrm{мкс.} \\
\tau_{0}=\frac{\tau_{\max }+\tau_{\min }}{2}=\frac{1,35+0,2}{2}=0,775 \mathrm{мкс} ; \\
\Delta \tau=\frac{\tau_{\max }-\tau_{\min }}{2}=\frac{1,35-0,2}{2}=0,575 \text { мкс. }
\end{gathered}
$$

Интервалы варьирования выбранных факторов определяем из табл. 3 .

Для вновь выбранных факторов составим матрицу плана и запишем её в табл. 4.
Таблица 3. Факторы и уровни их варьирования

Table 3. Factors and levels of their variation

Таблица 4. Матрица плана $2^{2}$

Table 4. Plan matrix $2^{2}$

\begin{tabular}{|c|c|c|c|c|c|}
\hline $\begin{array}{c}\text { № опыта } \\
\text { Experiment } \\
\text { no. }\end{array}$ & $x_{1}$ & $x_{2}$ & $\bar{y}_{i}=\bar{l}_{c 1 i}$ & $y_{i}^{*}=l_{c 1 i}^{*}$ & $S_{i}^{2}=\left(\bar{l}_{c 1 i}-l_{c 1 i}^{*}\right)^{2}$ \\
\hline 1 & -1 & -1 & 1,585 & 1,546 & 0,001521 \\
\hline 2 & +1 & -1 & 5,965 & 6,005 & 0,0016 \\
\hline 3 & -1 & +1 & 1,75 & 1,79 & 0,0016 \\
\hline 4 & +1 & +1 & 6,29 & 6,25 & 0,0016 \\
\hline
\end{tabular}

Формулы для расчёта коэффициентов уточнённого уравнения регрессии:

$$
\begin{gathered}
b_{0}=\frac{\sum_{i=1}^{N=4} \bar{y}_{i}}{N}=3,8975 ; \quad b_{1}=\frac{\sum_{i=1}^{N=4} \bar{y}_{i} x_{1 i}}{N}=2,23 ; \\
b_{2}=\frac{\sum_{i=1}^{N=4} \bar{y}_{i} x_{2 i}}{N}=0,1225 ; \\
\bar{l}_{c 1}^{*}=y^{*}=3,8975+2,23 x_{1}+0,1225 x_{2} .
\end{gathered}
$$

Оценку адекватности уравнения (16) осуществим по критерию Фишера. Для определения этого критерия найдем остаточную дисперсию по формуле

$$
S_{a d}^{2}=\frac{\left.\sum_{i=1}^{N=4} \overline{\left(y_{i}\right.}-y_{i}^{*}\right)^{2}}{f},
$$

где $f$ - число степеней свободы.

В нашем случае число степеней свободы $f=N-n-$ $1=N-k=4-3=1 ; N=2^{n}-$ общее число опытов в матрице (равно 4); $n$ - число факторов (равно 2); $k=3$ - число коэффициентов в уравнении. Величина остаточной дисперсии, определенная по выражению (17) равна

$$
S_{\text {ад }}^{2}=\frac{\sum_{i=1}^{N=4}\left(\bar{y}_{i}-y_{i}^{*}\right)^{2}}{f}=\frac{0,006321}{1}=0,006321 .
$$

Рассчитаем значение критерия Фишера по формуле:

$$
F=\frac{S_{\text {ад }}^{2}}{S_{\text {воспр }}^{2}} .
$$


Величина критерия, определенная по формуле (18), равна:

$$
F=\frac{S_{\text {ад }}^{2}}{S_{\text {воспр }}^{2}}=\frac{0,0063}{0,023}=0,275 .
$$

Табулированное значение критерия Фишера [11] для двух факторов при уровне значимости $p=0,05$ и числе степеней свободы 1 равно $F_{0,05}(n, f)=199,5$.

Так как выполняется неравенство $F_{005}(n, f)>F$, уравнение адекватно описывает эксперимент.

Поскольку во всех опытах контролировался один и тот же искусственно нанесённый дефект протяжённостью $l_{\text {д }}=0,5 \mathrm{MM}$, то систематическая погрешность в определении протяжённости дефекта определится из выражения:

$$
\Delta l=\bar{l}_{c 1}^{*}-0,5=3,3975+2,23 x_{1}+0,1225 x_{2} .
$$

В натуральных величинах уравнение (19) перепишется в виде:

$$
\begin{gathered}
\bar{l}_{c 1}^{*}=3,8975+2,23\left(\frac{U-1300}{700}\right)+0,1225\left(\frac{\tau-0,775}{0,575}\right)= \\
3,8975+0,003185714 U-4,1414+0,0213 \tau- \\
-0,165108695=0,409008695+0,003185714 U+0,0213 \tau . \\
\text { Окончательно получим: } \\
\bar{l}_{c 1}^{*}=-0,409008695+0,003185714 U+0,0213 \tau .(20)
\end{gathered}
$$

Переписав в натуральном масштабе выражение (19), с учетом выражения (20) получим

$$
\Delta l=-0,900900695+0,003185714 U+0,0213 \tau \text {. }
$$

Округлив все коэффициенты в уравнении (21), окончательно получим:

$$
\Delta l=\bar{l}_{c 1}^{*}-0,5=-0,901+0,00319 U+0,0213 \tau .
$$

\section{Практическая реализация результатов}

На рис. 3 приведена блок-схема измерителя дефектности, позволяющая реализовать полученные результаты.

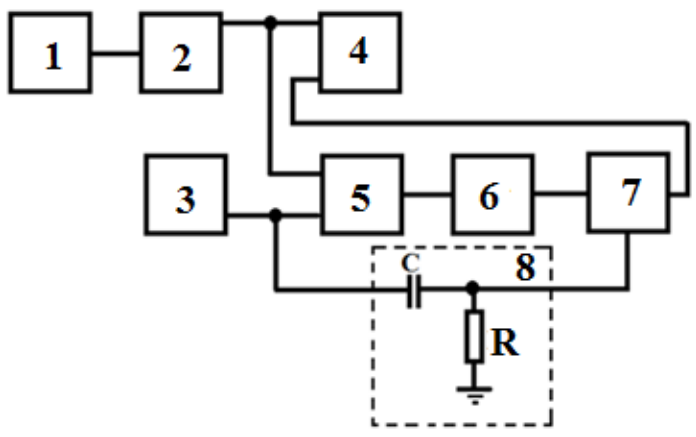

Puc. 2. Блок-схема измерителя дефектности: 1 - датчик скрости; 2 - формирователь импульсов; 3 датчик дефектов; 4 - счетчики мпульсов с входом разрешения счёта; 5 - ключевое устройство; 6 - счетчик с регулируемым коэффичиентом пересчета; 7 - триггер; 8 - дифференцирующая $R C$-цеепочка

Fig. 2. Block diagram of the defect meter: 1 -speed sensor; 2 - pulse shaper; 3 - defect sensor; 4 - pulse counter with counting resolution input; 5 - key device; 6 - counter with adjustable conversion factor; 7 -trigger; 8 -differentiating $R C$-chain
Конструкция датчика дефектов представлена на рис. 3.

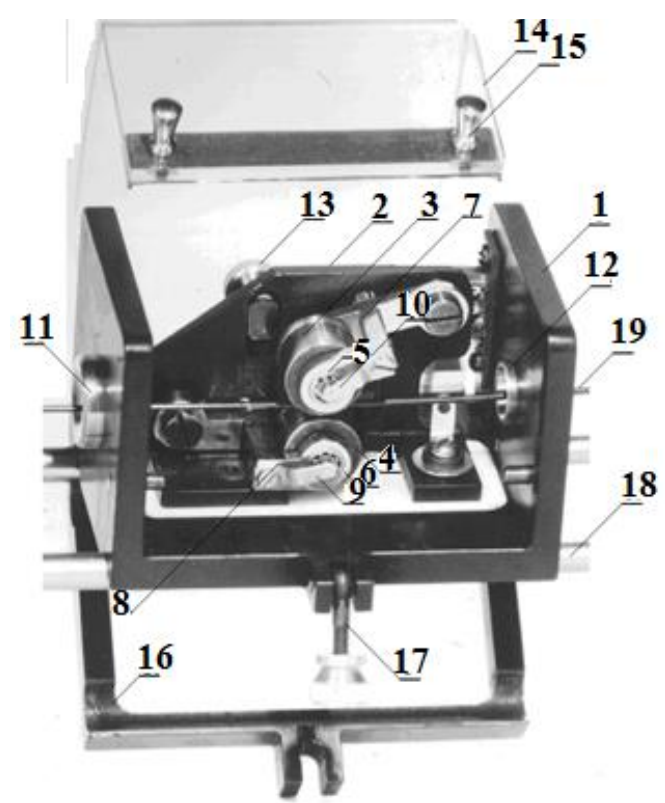

Pис. 3. Датчик дефектов: 1-каркас; 2 - платформа; 3, 4 - дискообразные электроды; 5, 6 - шарикоподшипники; 7, 8-коромысла; 9, 10 - контакты скользящие; 11, 12 - втулки направляющчие; 13 концевой выключатель; 14 - зашитная крышка; 15 - фиксаторы; 16 - платформа; 17 - крепежный элемент датчика; 18 - держатель; 19 провод

Fig. 3. Defects sensor: 1 -frame; 2 -platform; 3, 4-discshaped electrodes; 5, 6-balls; 7, 8-arms; 9, $10-$ sliding contacts; 11, 12 - bushing guides; 13 switch; 14 - protective cover; 15 - pins; 16 platform; 17- mounting element of sensor; 18 holder; 19 -wire

Измерение протяженности дефектов осуществляется с помощью датчика скорости, приведённого на рис. 4.

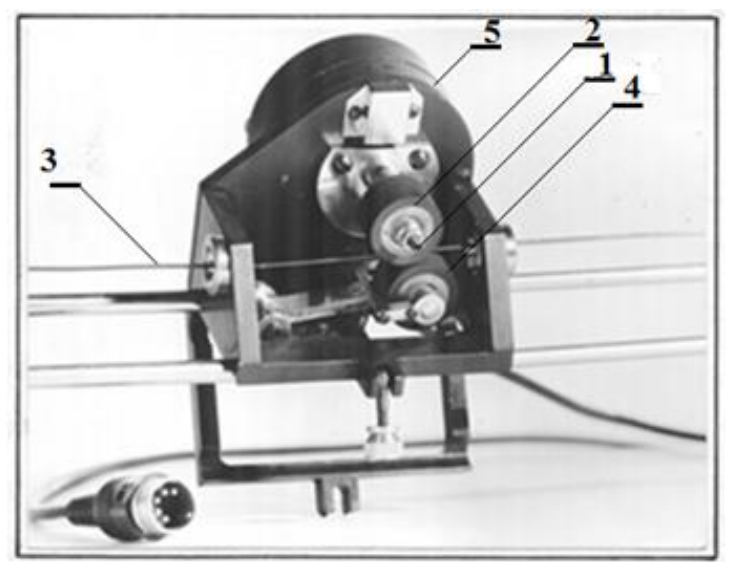

Pис. 4. Датчик скорости: 1 - ось; 2 - ведущчий ролик; 3 - изолированый провод; 4 - свободно вращқающийся ролик; 4 - растровый блок

Fig. 4. Speed sensor: 1 - axis; 2 - drive roller; 3 insulated wire; 4 -free rotating roller; 4 -bit block 
В основу датчика скорости положен принцип фотоэлектрического преобразователя перемещений, который реализуется следующим образом. В датчике скорости находится измерительный растр, насаженный на одну ось -1 с ведущим прорезиненным роликом - 2. Изолированный провод - 3 в процессе контроля прижимается к образующей поверхности ведущего ролика - 2 другим, подпружиненным, свободно вращающимся роликом - 4. Измерительный растр представляет собой диск из непрозрачного материала с равномерно расположенными по длине окружности радиальными прорезями. За измерительным растром находится индикаторный растр. По обеим сторонам индикаторного и измерительного растров на уровне прорезей расположен источник света и фотоприемник. При движении провода приходит во вращение ведущий ролик, и вместе с ним измерительный растр. Происходит модуляция светового потока, поступающего на фотоприемник. При этом освещённость фотоприёмника максимальна при совмещении прорезей измерительного и индикаторного растров, и минимальна, когда прорези одного растра закрываются не прорезанными участками другого растра. Полный цикл изменения освещённости происходит при перемещении растра на величину шага. Электрическая схема преобразует изменение освещённости фотоприёмника в электрические импульсы. За период следования одного импульса через датчик скорости пройдёт участок провода, протяженностью $l_{c}=\frac{\pi D}{M}$, где $D$ - диаметр ведущего ролика датчика скорости; $M$ - количество прорезей измерительного растра.

Точность определения продольного размера дефектов возрастает при снижении величины $l_{c}$. Снижение значения $l_{c}$ можно добиться либо уменьшением $D$ ролика, либо увеличением количества щелей $M$. Уменьшение величины $D$ лимитировано возрастанием трения между изоляцией провода и поверхностью ролика при повышенных скоростях протягивания через датчик провода, при этом возможно возникновение проскальзывания провода. Повышение количества прорезей при ограниченных размерах датчика приводит к необходимости снижения размеров этих прорезей. Нам удалось при диаметре ролика 10,1 мм изготовить растр, имеющий 240 прорезей. Это дало возможность уменьшить величину $l_{c}$ до значения 0,25 мм. При величине $l_{c}=0,25$ мм нельзя достичь низкой погрешности измерения протяженности дефекта, так как ошибка с зоны $t_{c}$ может давать \pm 1 импульс. Это приводит к погрешности измерения дефекта $\pm 0,25$ мм. Для уменьшения ошибок в прибор введен умножитель частоты с коэффициентом умножения $k=10$. На вход умножителя частоты поступают импульсы с датчика скорости. С момента прихода переднего фронта импульса осуществляется счет импульсов $n_{1}$ с тактового генератора частотой $f_{1}=2$ МГц. Счёт импульсов осуществляется до прихода следующего переднего фронта импульса с датчика скорости. Счетчик обнуляется и начинает счёт следующих $n_{2}$ импульсов. Поступившие на вход схемы 2 (рис. 2) $n_{1}$ импульса вычитаются с частотой $f_{2}=20$ МГц. После того, как произойдёт вычитание последнего импульса, на выходе схемы 2 генерируется импульс скорости, и в ней вновь начинается вычитание $n_{1}$ импульсов с $f_{2}=20$ МГц. Указанная процедура повторяется до момента прихода на вход схемы пачки новых $n_{2}$ импульсов. При этом каждый импульс на выходе схемы 2 инициирует появление $k=\frac{f_{2}}{f_{1}}$ импульсов скорости. За время одного сформированного импульса скорости провод переместится на элементарное расстояние $l_{3}=\frac{\pi D}{k M}=\frac{0,25}{10}=0,025$ мм.

Контроль дефектности изоляции провода производят следующим образом. При прохождении через датьчик скорости - 1 контролируемого провода счетчик генерирует серию импульсов, частота следования которых прямопропорциональна скорости движения провода. Сформированная пачка импульсов поступает на вход умножителя частоты, которая изменяется пропорционально скорости провода. Эта пропорциональность обеспечивает постоянность величины $l_{3}$, определяемой по прведенной выше формуле, не зависимо от того как изменилась скорость провода.

При подходе зоны дефектного участка изоляции к первичному преобразователю дефектов - 3 возникающим в преобразователе сигналом запускается ключевое устройство - 5 , пропускающее на счётчик -6 импульсы скорости. Для устранения систематической погрешности при контроле протяженности дефектов в счетчике - 6 введен коэффициент пересчета. Величина этого коффициента $m$ оценивается по формуле $m=\frac{\Delta l}{l_{s}}$. В табл. 5 приведена экспериментальная и расчетная по формуле (22) систематическая погрешность и коэффициент пересчёта в счетчике -6 .

Таблица 5. Погрешность и коэффиииент пересчета Table 5. Error and conversion factor

\begin{tabular}{|c|c|c|c|c|c|}
\hline 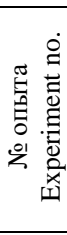 & $x_{1}$ & $x_{2}$ & $\begin{array}{c}\text { Эксперимен- } \\
\text { тальная } \\
\text { систематическая } \\
\text { погрешность, } \Delta l_{\text {э }} \\
\text { Experimental } \\
\text { systematic error, } \\
\Delta l_{3} \\
\end{array}$ & \begin{tabular}{|l} 
Систематическая \\
погрешность по \\
формуле (22), $\Delta l_{\mathrm{p}}$ \\
Systematic \\
sinfulness \\
according to the \\
formula $(22), \Delta l_{\mathrm{p}}$ \\
\end{tabular} & $\begin{array}{l}\text { Коэффи- } \\
\text { циент } \\
\text { пересче- } \\
\text { та, } m \\
\text { Conversion } \\
\text { factor, } m\end{array}$ \\
\hline 1 & 600 & 0,2 & 1,005 & 1,046 & 41,84 \\
\hline 2 & 2000 & 0,2 & 5,465 & 5,505 & 220,2 \\
\hline 3 & 600 & 1,35 & 1,25 & 1,29 & 51,6 \\
\hline 4 & 2000 & 1,35 & 5,79 & 5,75 & 230 \\
\hline
\end{tabular}

Разрешение на счет счетчика протяженности дефектов устанавливается после прихода $m$-го импульса в соответствии с табл. 5 .

\section{Выводы}

Проведен анализ особенности характеристик газоразрядного датчика дефектов в изоляции обмоточных проводов. Выявлены параметры разрядной цепи первичного преобразователя, при которых может происходить смена вида разряда в первичном преобразова- 
теле. Установлено, что наиболее приемлемым видом разряда при контроле является коронный. Выделена стабильная зона этого разряда, из которой можно извлечь полезную информацию о протяженности дефектного участка изоляции. С помощью корреляционного анализа установлено, что скорости движения провода, при которых осуществляется намотка обмоток в реальных технологических процессах, не влияют на длительность стабильной зоны сигнала с дефекта и на точность определения протяженности дефектов по указанной зоне. Проведены исследова-

\section{СПИСОК ЛИТЕРАТУРЫ}

1. ГОСТ IEC 60851-5-2011. Провода обмоточные. Методы испытаний. Ч. 5. Электрические свойства. - М.: Стандартинформ, 2013. - $10 \mathrm{c}$.

2. Дудкин А.Н., Леонов А.П., Супуева А.С. Оценка влияния уровня дефектности эмалированных проводов на надежность межвитковой изоляции // Доклады ТУСУРа. - 2017. - Т. 20. № 2. - C. 123-126.

3. Смирнов Г.В. Пооперационный контроль процесса изготовления обмоток электрических машин - М.: Научно-техническое изд-во «Горячая линия - Телеком», 2018. - 156 с.

4. Курбатова О.А., Павлюченко В.М. Монтаж и ремонт горных машин и электрооборудования. - Владивосток: Изд-во ДВГТУ, 2004. - $286 \mathrm{c}$

5. Колмаков Е.А., Кондрашов П.М., Зеньков И.В. Обзор конструкций фильтров в составе погружных электроцентробежных насосов при добыче нефти // Вестник КузГТУ. - 2016. № 1. - C. $150-155$.

6. Компьютерная система для функциональной диагностики электроприводов карьерных экскаваторов // В.Г. Каширских, А.Н. Гаргаев, В.М. Завьялов, И.Ю. Семыкина // Вестник КузГТУ. - 2016. - № 6. - С. 159-168.

7. Русский Е.Ю. Анализ прочности роторов шахтных осевых вентиляторов // Вестник КузГТУ. - 2015. - № 2. - С. 31-34.

8. Герике П.Б. Определение дефектов динамического оборудования тяговых лебедок экскаваторов типа драглайн по параметрам механических колебаний // Вестник КузГТУ. - 2014. № 1. - C. 21-26.

9. Смирнов Г.В., Ведяшкин М.В. Контроль пропитки - эффективный фактор повышения надежности электродвигателей горнодобывающей, строительной и других отраслей промышленности // Известия Томского политехнического университета. Инжиниринг георесурсов. - 2019. - Т. 330. - № 1. - С. 51-64.

10. Анализ надёжности электродвигателей, используемых в современных электроприводах / А.Ф. Бурков, Е.В. Катаев, Г.Е. Кувшинов, К.В. Чупина // Электроника и электротехника. - 2017. - № 1. - С. 1-6.

11. Смирнов Г.В. Моделирование и оптимизация объектов и процессов. - М.: Научно-техническое изд-во «Горячая линия Телеком», 2018. - 176 c. URL: http://www.techbook.ru/ book.php?id_book=1045 (дата обращения: 15.02.2020).

12. Smirnov G.V.. ., Smirnov D.G. Nondestructive testing for flaws in the insulation of winding wires // Russian Journal of Nondestructive Testing. - 2016. - V. 52. - № 8. - P. 469-477. ния с использованием теории планирования эксперимента, и построено адекватное уравнение регрессии, показывающее зависимость систематической погрешности определения протяженности дефектного участка изоляции провода от параметров разрядной цепи датчика дефектов. Показано, что указанная систематическая погрешность в определении протяженности дефектов линейно зависит от напряжения контроля на датчике дефектов и постоянной времени разрядной цепи этого датчика.

13. Smirnov G., Kovalsky A. Primary gas discharge transducer and its application // MATEC Web of Conferences. - 2018. - P 143. URL: https://doi.org/10.1051/matecconf/201814303002 .

14. Смирнов Г.В., Смирнов Д.Г. Физические основы неразрушающего контроля изоляции обмоток электроприводов горношахтного и нефтепромыслового оборудования // Известия Томского политехнического университета. Инжиниринг георесурсов. - 2016. - Т. 327. - № 2. - C.102-116.

15. PDIV characteristics of twisted-pair of magnet wires with repetitive impulse voltage / K. Kimura, S. Ushirone, T. Koyanagi, M. Hikita // IEEE Trans. on dielectrics and electrical Ins. - June 2007. - V. 14. - № 3. - P. 744-750.

16. A new generation of aluminum enameled winding wires / T. Knych, A. Mamala, M. Jablonski, P. Uliasz // Wire Journal International. - 2012. - V. 45. - № 1. - P. 55-61.

17. Райзер Ю.П. Физика газового разряда. - М.: Наука, 1987. $592 \mathrm{c}$.

18. Баранов В.Ю., Напартович А.П., Старостин А.Н. Тлеющий разряд в газах повышенного давления // Физика плазмы. 1984. - Т. 10. - № 5. - С. 90-97.

19. Королев Ю.Д., Месяц Г.А. Автоэмиссионные и взрывные процессы в газовом разряде. - Новосибирск: Наука, 1982. $255 \mathrm{c}$.

20. Baldanov B.B., Ranzhurov Ts.V. Modification of the PTFE film in low-current argon discharges // High-Tech in Chemical Engineering-2014: XV International Scientific Conference. Zvenigorod, 2014. - P. 242-247.

21. Gerling T. et al. Influence of the capillary on the ignition of the transient spark discharge // J. Phys. D: Appl. Phys. - 2013. V. 46. - P. 145-150.

22. Kogelschatz U. Dielectric-barrier discharges: their history, discharge physics, and industrial application // Plasma chemistry and plasma processing. - 2003. - V. 23. - № 1. - P. 1-16.

23. He S.J., Jing H. Spatio-temporal characteristics of Trichel pulse at low pressure // Physics Plasmas. - 2014. - V. 21 - P. 012112.

24. Trichel pulses in a negative corona discharge in air at low pressure / He Shou Jie, Ha Jing, Han Yu Honq, Li Zhi Oifnq // Journal of Physics: Conference Series. - 2013. - V. 418. - P. 012091.

25. Ишханян М.В., Карпенко Н.В. Эконометрика. Ч. 1. Парная регрессия. - М.: Московский государственный университет путей сообщения, 2016. -117 с.

Поступила 22.02.2020 2.

\section{Информация об авторах}

Смирнов Г.В., доктор технических наук, профессор, директор Научно-исследовательского института электронного технологического оборудования и систем связи Томского государственного университета систем управления и радиоэлектроники. 


\title{
UDC 658.382 .3
}

\section{CONTROL OVER WINDING INSULATION DEFECTS - THE EFFECTIVE WAY TO IMPROVE RELIABILITY OF ELECTRIC MOTORS IN MINING, CONSTRUCTION AND OTHER INDUSTRIES}

\author{
Gennady V. Smirnov, \\ smirnov@main.tusur.ru \\ Tomsk State University of Control Systems and Radioelectronics, \\ 40, Lenin Avenue, Tomsk, 634045, Russia.
}

\begin{abstract}
The relevance of the work. Such spheres of human activity, as geological exploration, production, transportation and recycling use the means of mechanization and automation with application of different types of electric drives. The reliability of these devices is determined by winding insulation quality. In this regard, the search for effective means of controlling wire enamel insulation, improving the insulation quality, eliminating technological reasons of defect occurrence are popular and topical.

The aim of the work is to research the influence of defect meter parameters, modes of control and gas-discharge defects flowing in a primary converter on accuracy of measuring the geometrical sizes of defects in wire insulation.

Methods: oscilloscope, micrometer, theory of planning the experiments, methods of correlation estimates between the control parameters and regression analysis.

Results. The author has revealed and stated in the paper the regularities of occurrence of systematic error in measuring defect extent when controlling wire isolation at winding, by the defectiveness meter using the gas-discharge sensor as the primary defect converter. It is shown that two factors: control $U$ voltage on the defect gas-discharge sensor and the time constant $T=R C$ of the sensor discharge circuit, influence most of all the value of the specified errors. Using the correlation and regression analyses the adequate model of systematic error was constructed; it was established that the wire motion velocity has no significant effect on the magnitude of this error. When applying the theory of planning the experiments the author obtained the adequate equation based on bias from the control settings. The paper demonstrates the practical application of the established patterns to improve the accuracy of controlling the defect extent in wire insulation in gas sensor meters.
\end{abstract}

\section{Key words:}

Control, defect, length, primary converter of defects, error, speed sensor, voltage, time constant, low-voltage winding, corona discharge.

\section{REFERENCES}

1. GOST IEC 60851-5-2011. Provoda obmotochnye. Metodika Ispytaniya. Ch. 5. Elektritchseskie svoystva [State Standard 60851-5-2011. Winding wires. Test methods. P. 5. Electrical properties]. Moscow, Standartinform Publ., 2013. 10 p.

2. Dudkin A.N., Leonov A.P., Supoena A.S. Assessment of the impact of the level of defect of enameled wires on the reliability of inter-turn insulation. Reports Tomsk State University of Control Systems and Radioelectronics, 2017, vol. 20, no. 2, pp. 123-126. In Rus.

3. Smirnov G. V. Pooperatsionny kontrol protsessa izgotovleniya obmotok elektritcheskikh mashin [Operational control of manufacturing windings of electric machines]. Moscow, Hot line - Telecom Publ. house, 2018. 156 p.

4. Kurbatov O.A., Pavlyuchenko V.M. Montazh i remont gornykh mashin i elektrooborudovaniya [Installation and repairing of mining machinery and electrical devices]. Vladivostok, Far Eastern State Technical University Publ. house, 2004. 286 p.

5. Kolmakov E.A., Kondrashov P.M., Zenkov I.V. Review of filter design in the composition of electrical submersible pumps in oil production. Bulletin of the Kuznetsky Technical University, 2016, no. 1, pp. 150-155. In Rus.

6. Kashira A.N., Garaev V.M., Zavyalov I.Yu., Semykina I.Yu. Computer system for functional diagnostics of electric drives of quarrytion excavators. Bulletin of the Kuznetsky Technical University, 2016, no. 6, pp. 159-168. In Rus.

7. Russian E.Y. The Analysis of the strength of the rotor shaft of axial fans. Bulletin of the Kuznetsky Technical University, 2015, no. 2, pp. 31-34. In Rus.

8. Gerik P. B. Defining defects of dynamic equipment, traction hoists excavators of the dragline type in the parameters of mechanical vibrations. Bulletin of the Kuznetsky Technical University, 2014, no. 1, pp. 21-26. In Rus.

9. Smirnov G.V., Vedyashkin M.V. Impregnation control is an effective factor in improving the reliability of electric motors in mining, construction and other industries. Bulletin of the Tomsk Polytechnic University. Geo Assets Engineering, 2019, vol. 330, no. 1, pp. 51-64. In Rus.
10. Burkov A.F., Kataev E.V., Kuvshinov G.E., Chupina K.V. Reliability analysis of electric motors used in modern electric drives. Electronics and electrical engineering, 2017, no. 1, pp. 1-6. In Rus.

11. Smirnov G.V. Modelirovanie i optimisaziya obektov i protsessov [Modeling and optimization of objects and processes]. Moscow, Hot line - Telecom Publ. house, 2018. 176 p. Available at: http://www.techbook.ru/book.php?id_book=1045 (accessed 15 February 2020).

12. Smirnov G.V., Smirnov D.G. Nondestructive testing for flaws in the insulation of winding Wires. Russian Journal of Nondestructive Testing, 2016, vol. 52, no. 8, pp. 469-477.

13. Smirnov G., Kovalsky A. Primary gas discharge transducer and its application. MATEC Web of Conferences, 2018, pp. 143. Available at: https://doi.org/10.1051/matecconf/201814303002 (accessed 15 February 2020).

14. Smirnov G.V., Smirnov D.G. Physical basis of non-destructive control of winding insulation of electric drives of mining and oil field equipment. Bulletin of the Tomsk Polytechnic University. Geo Assets Engineering, 2016, vol. 327, no. 2, pp. 102-116. In Rus.

15. Kimura K., Ushirone S., Koyanagi T., Hikita M. PDIV characteristics of twisted-pair of magnet wires with repetitive impulse voltage. IEEE Trans. on dielectrics and electrical Ins., June 2007, vol. 14, no. 3, pp. 744-750.

16. Knych T., Mamala A., Jablonski M., Uliasz P. A new generation of aluminum enameled winding wires. Wire Journal International, 2012, vol. 45, no. 1, pp. 55-61.

17. Raizer Yu.P. Fizika gazovogo razryada [Physics of gas discharge.] Moscow, Nauka Publ., 1987. 592 p.

18. Baranov V.Yu., Napartovich A.P., Starostin A.N. Tleyushchy razryad $\mathrm{v}$ gazakh povyschennogo davleniya [Glow discharge in high-pressure gases]. Plasma physics, 1984, vol. 5, pp. 90-97.

19. Korolev Yu.D., Mesyats G.A. Avtoemissionnye $i$ vzryvnye protsessy $v$ gazovom razryade [Field emission and explosive processes in gas discharge]. Novosibirsk, Nauka Publ., 1982. 255 p.

20. Baldanov B.B., Ranzhurov Ts.V. Modification of the PTFE film in low-current argon discharges. XV International Scientific Conference. High-Tech in Chemical Engineering-2014. Zvenigorod, 2014. pp. 242-247. 
21. Gerling $\mathrm{T}$. Influence of the capillary on the ignition of the transient spark discharge. J. Phys. D: Appl. Phys., 2013, vol. 46, pp. 145-150.

22. Kogelschatz U. Dielectric-barrier discharges: their history, discharge physics, and industrial application. Plasma chemistry and plasma processing, 2003, vol. 23, no. 1, pp. 1-16.

23. He S.J., H. Jing Spatio-temporal characteristics of Trichel pulse at low pressure. Physics Plasmas, 2014, vol. 21, pp. 012112.
24. He Shou Jie, Ha Jing, Han Yu Honq, Li Zhi Oifnq. Trichel pulses in a negative corona discharge in air at low pressure. Journal of Physics: Conference Series, 2013, vol. 418, pp. 012091.

25. Ishkhanyan M.V., Karpenko N.V. Ekonometrika. Ch. 1. Parnaya regressiya [Econometrics. P. 1. Pair regression]. Moscow, Moscow state railway University Publ., 2016. 117 p.

Received: 22 February 2020.

\section{Information about the authors}

Gennady V. Smirnov, Dr. Sc., professor, director of the Research Institute of electronic technological equipment and communication systems, Tomsk State University of Control Systems and Radioelectronics. 